\title{
Faraday conversion and magneto-ionic variations in fast radio bursts
}

\author{
H. K. Vedantham ${ }^{\oplus 1,2 \star}$ and V. Ravi ${ }^{3}$ \\ ${ }^{1}$ ASTRON, Netherlands Institute for Radio Astronomy, Oude Hoogeveensedijk 4, NL-7991 PD Dwingeloo, the Netherlands \\ ${ }^{2}$ Kapteyn Astronomical Institute, University of Groningen, PO Box 800, NL-9700 AV Groningen, the Netherlands \\ ${ }^{3}$ Harvard-Smithsonian Center for Astrophysics, 60 Garden Street, Cambridge, MA 02138, USA
}

Accepted 2019 March 7. Received 2019 March 7; in original form 2019 January 16

\begin{abstract}
The extreme, time-variable Faraday rotation observed in the repeating fast radio burst (FRB) 121102 and its associated persistent synchrotron source demonstrates that some FRBs originate in dense, dynamic, and possibly relativistic magneto-ionic environments. Besides rotation of the linear polarization vector (Faraday rotation), such media can generally convert linear to circular polarization (Faraday conversion). We use non-detection of Faraday conversion, and the temporal variation in Faraday rotation and dispersion in bursts from FRB 121102 to constrain models where the progenitor inflates a relativistic nebula (persistent source) confined by a cold dense medium (e.g. supernova ejecta). We find that the persistent synchrotron source, if composed of an electron-proton plasma, must be an admixture of relativistic and non-relativistic (Lorentz factor $\gamma<5$ ) electrons. Furthermore, we independently constrain the magnetic field in the cold confining medium, which provides the Faraday rotation, to be between 10 and $30 \mathrm{mG}$. This value is close to the equipartition magnetic field of the confined persistent source implying a self-consistent and overconstrained model that can explain the observations.
\end{abstract}

Key words: polarization-radiative transfer-radio continuum: general.

\section{INTRODUCTION}

By virtue of their large Faraday rotation, at least two fast radio burst (FRB) sources (FRB 121102 and FRB 110523; Spitler et al. 2014; Masui et al. 2015) are observed to reside in dense magneto-ionic environments. FRB 121102 is also co-located with a relativistic synchrotron-emitting nebula (Marcote et al. 2017). Propagation through a magneto-ionic medium leads to the well-known Faraday rotation effect. In addition to Faraday rotation, propagation through magneto-ionic media can also lead to Faraday conversion, wherein linearly polarized light is converted to circularly polarized light and vice versa. Faraday conversion is a relatively weak effect. However, observable levels of conversion can occur in two astrophysical scenarios: (i) when the magneto-ionic medium is mildly relativistic (Lorentz factor $\gamma \gtrsim 3$ typically; see e.g. Sazonov 1969; Pacholczyk \& Swihart 1970; Huang \& Shcherbakov 2011), or (ii) when the magnetic field is large ( $B \sim 1 \mathrm{G}$ typically for $\mathrm{cm}$-wavelength observations) and the angle between the wave vector and the magnetic field is almost exactly $\pi / 2$ (so-called quasi-transverse propagation; see e.g. Cohen 1960; Zheleznyakov \& Zlotnik 1964). Case (i) is thought to be pertinent to circular polarization in some active galactic nuclei jets (Homan, Attridge \& Wardle 2001), whereas case (ii) is commonly encountered in propagation through stellar corona (White, Thejappa \& Kundu 1992). We exclusively deal with

^E-mail: vedantham@astron.nl case (i) here and refer the reader to Gruzinov \& Levin (2019) for a discussion on the effects of quasi-transverse propagation FRBs.

In this Letter, by taking FRB 121102 as a test case, we argue for the first time that Faraday conversion is an observable effect in some FRBs and leads to upper limits on the circumburst magnetic field and the density of low Lorentz factor electrons $(3 \lesssim \gamma \lesssim 100)$ that are otherwise observationally inaccessible. We show that additional constraints from temporal variations in the dispersion and Faraday rotation of FRB 121102 critically constrain proposed models for the environment of FRB 121102.

In Section 2, we summarize existing observations and modelindependent constraints on the magneto-ionic environment of FRB 121102 and its associated persistent radio source. In Section 3, we describe the additional constraints implied by the non-detection of circular polarization in FRB 121102, given predictions from Faraday conversion. We discuss the implications of these results for models where FRB 121102 is located within the persistent radio source in Section 4, and conclude in Section 5.

\section{GENERAL CONSTRAINTS ON THE ENVIRONMENT OF FRB 121102}

\subsection{Observations}

We first summarize the known properties of the magneto-ionic environment surrounding the repeating FRB 121102 (Spitler et al. 2016). It has been localized to an H II region in a galaxy at a 
redshift $z=0.19$ (luminosity and angular diameter distances of $d_{\mathrm{L}} \approx 970 \mathrm{Mpc}$ and $d_{\mathrm{A}} \approx 680 \mathrm{Mpc}$, respectively; Chatterjee et al. 2017). Additionally, the FRB 121102 bursts are (i) co-located to within $40 \mathrm{pc}$ ( 95 per cent confidence) with a persistent flatspectrum $(1-10 \mathrm{GHz})$ radio source with flux density $S_{v} \approx 200 \mu \mathrm{Jy}$ at $3 \mathrm{GHz}$ (Marcote et al. 2017), and (ii) show very high levels of Faraday rotation, quantified by the rotation measure (RM), ${ }^{1}$ $\mathrm{RM}=1.46 \times 10^{5} \mathrm{rad} \mathrm{m}^{-2}$ in the source frame. The RM reduced by $\sim 10$ per cent in 7 months (Michilli et al. 2018), whereas its dispersion measure (DM) $)^{2}$ increased by just $1-3 \mathrm{pc} \mathrm{cm}^{-3}$ in $4 \mathrm{yr}$ (Hessels et al. 2018). Finally, the bursts are nearly 100 per cent linearly polarized between 4 and $8 \mathrm{GHz}$; the circularly polarized fraction (Stokes $V / I$ ) is below a few per cent (Michilli et al. 2018). We will use $V / I<2$ per cent in this Letter.

\subsection{Dispersion and Faraday rotation}

The DM in the entire host galaxy is constrained to be less than $250 \mathrm{pc} \mathrm{cm}^{-3}$ (Tendulkar et al. 2017). If an amount, $\mathrm{DM}_{\mathrm{RM}}$, of that is in the Faraday-rotating nebula, Hessels et al. (2018) obtain the following bound by requiring the magneto-ionic medium to be transparent to free-free absorption at the lowest frequency at which bursts have been observed ( $1 \mathrm{GHz})$ :

$T_{4}^{2.3} \mathrm{DM}_{\mathrm{RM}}>150\left(\frac{\beta}{\eta_{B}^{2}}\right)$,

where $T_{4}$ is the gas temperature in units of $10^{4} \mathrm{~K}, \beta$ is the ratio of thermal to magnetic pressure, and $\eta_{B} \leq 1$ is a geometric factor equal to the mean value of $\cos \theta$ along the propagation path in the Faradayrotating medium, where $\theta$ is the angle between the propagation direction and the ambient magnetic field. For an ordered field, we have $\eta_{B}=\cos \theta$. Significantly smaller values must be expected for a highly tangled field due to partial cancellation of positive and negative Faraday rotation. Further, for any given choice of $\mathrm{DM}_{\mathrm{RM}}$, the corresponding magnetic field that can generate the observed Faraday rotation is

$B=\frac{0.18}{\mathrm{DM}_{\mathrm{RM}} \eta_{B}} \mathrm{G}$

We now address the RM variations using two generic models for the magneto-ionic medium. We will address specific models in Section 4.

Expanding nebula. If the observed RM decrease is due to the expansion of a nebula of radius $R$ (e.g. Waxman 2017; Margalit \& Metzger 2018), then $\mathrm{DM}_{\mathrm{RM}} \propto R^{-2}$ and $B \propto R^{-1.5}$, maintaining the same plasma $\beta$ and geometric factor $\eta_{B}$. We therefore have $\Delta \mathrm{RM} / \mathrm{RM}=\Delta B / B+\Delta \mathrm{DM}_{\mathrm{RM}} / \mathrm{DM}_{\mathrm{RM}}$, where (to first order) $\Delta B / B=-1.5 \Delta R / R$, and $\Delta \mathrm{DM}_{\mathrm{RM}} / \mathrm{DM}_{\mathrm{RM}}=-2 \Delta R / R$. The observed 10 per cent variation in $\mathrm{RM}$ over 7 months then implies $\Delta R / R \approx 0.05 \mathrm{yr}^{-1}$ and the nebula is therefore expanding on a time-scale of about $\tau \approx 20 \mathrm{yr}$. The implied -5.8 per cent decrease in $\mathrm{DM}_{\mathrm{RM}}$ due to the expansion must be insignificant compared to the observed increase of $1-3 \mathrm{pc} \mathrm{cm}^{-3}$ in the total DM over a longer time-scale than the reported RM variations; the observed DM variations presumably occur in plasma unrelated

\footnotetext{
${ }^{1}$ The plane of linear polarization rotates by an angle $\mathrm{RM} \lambda^{2} ; \mathrm{RM}=$ $0.812 \int\left(B_{\|} / \mu \mathrm{G}\right)\left(n_{\mathrm{e}} / \mathrm{cm}^{-3}\right)(\mathrm{d} s / \mathrm{pc}) \mathrm{rad} \mathrm{m}^{-2}$, where $\mathrm{d} s$ is the line element, $B_{\|}$is the line-of-sight component of the magnetic field, and $n_{\mathrm{e}}$ is the free electron density.

${ }^{2}$ The dispersion measure is the column density of free electrons towards the source, usually given in units of $\mathrm{pc}^{-3}$.
}

to the Faraday-rotating plasma. Hence, we can safely place the constraint $\mathrm{DM}_{\mathrm{RM}}<17.5 \mathrm{pc} \mathrm{cm}^{-3}$. These constraints do not differ significantly for other expansion scenarios. For instance, adiabatic expansion of a tangled field $\left(B \propto R^{-2}\right.$ ) gives $\tau \approx 23.33 \mathrm{yr}$ and $\mathrm{DM}_{\mathrm{RM}}<20 \mathrm{pc} \mathrm{cm}^{-3}$.

Transverse motion. Alternatively, the RM variations could be due to transverse motion of the FRB source across a nebula over a characteristic time-scale of $\tau \approx 5.83 \mathrm{yr}$. If we assume the line-of-sight extent of the Faraday-rotating nebula to be roughly equal to its transverse scale length, we have $\Delta \mathrm{RM} / \mathrm{RM} \approx \Delta \mathrm{DM}_{\mathrm{RM}} / \mathrm{DM}_{\mathrm{RM}} \approx 0.1$. The lack of significant DM variations accompanying the $R M$ variations now gives a more stringent constraint of $\mathrm{DM}_{\mathrm{RM}}<10 \mathrm{pc} \mathrm{cm}^{-3}$.

Using the formalism from section 4.4 of Hessels et al. (2018), we can translate the above limits on $\mathrm{DM}_{\mathrm{RM}}$ to the underlying plasma parameters. In doing so, we use the following notation: (a) $\mathrm{DM}_{10}=$ $\mathrm{DM}_{\mathrm{RM}} /\left(10 \mathrm{pc} \mathrm{cm}^{-3}\right)$, and (b) the parameter values for the expanding nebula and transverse motion scenarios are given in parentheses, respectively. With this notation, the limits are

$$
\begin{aligned}
\mathrm{DM}_{10} & <(1.75,1.0), \\
T_{4} & >2.72\left(\frac{\beta}{\eta_{B}^{2}}\right)^{1 / 2.3} \mathrm{DM}_{10}^{1 / 2.3}, \\
B & =18 /\left(\mathrm{DM}_{10} \eta_{B}\right) \mathrm{mG}, \\
n_{\mathrm{e}} & =9.3 \times 10^{6}\left(\frac{\eta_{B}^{2} T_{4}}{\beta}\right)^{-1} \mathrm{DM}_{10}^{-2} \mathrm{~cm}^{-3}, \\
l & =1.1 \times 10^{-6}\left(\frac{\eta_{B}^{2} T_{4}}{\beta}\right) \mathrm{DM}_{10}^{3} \mathrm{pc}, \\
\tau & =(20,5.83) \mathrm{yr}, \\
v & =(0.054,0.185)\left(\frac{\eta_{B}^{2} T_{4}}{\beta}\right) \mathrm{DM}_{10}^{3} \mathrm{~km} \mathrm{~s}^{-1} .
\end{aligned}
$$

The velocity, $v$, in the two cases must be interpreted as the radial expansion speed of the nebula and transverse speed of the source with respect to the Faraday-rotating medium, respectively. We will return to these constrains with regards to specific models in Section 4.

\subsection{The persistent radio source}

The properties of the persistent radio source associated with FRB 121102 may be constrained independently of the Faradayrotating medium. We assume equipartition between the relativistic gas and magnetic field as is common in synchrotron sources ${ }^{3}$ (Readhead 1994). The source becomes self-absorbed at $1.5 \mathrm{GHz}$ for radius $R_{\text {per }}<0.05 \mathrm{pc}$; this is thus the lower bound on the source size. European Very Long Baseline Interferometry (VLBI) Network observations of the source at $5 \mathrm{GHz}$ set an upper bound on the source radius of $R_{\text {per }} \lesssim 0.35$ pc (Marcote et al. 2017). This is consistent with the $\approx 30$ percent amplitude modulations observed in the source at $3 \mathrm{GHz}$ (Chatterjee et al. 2017) being caused by refractive interstellar scintillation in the Milky Way interstellar medium (ISM; Walker 1998). For any radius within the allowed range $\left(0.05<R_{\mathrm{per}} / \mathrm{pc}<0.35\right)$, we can determine the equipartition magnetic field, $B_{\text {eq }}$, and the column of relativistic electrons, $N_{\text {rel }}$, using the standard expressions for synchrotron emissivity and absorption coefficients (Rybicki \& Lightman 1979,

\footnotetext{
${ }^{3}$ In the model of Waxman (2017), which we discuss in Section 4.1, equipartition is required by dynamical and source-size (from scintillation) constraints.
} 
their equations 6.36 and 6.53). We assume a power-law energy distribution of radiating electrons with somewhat shallow index of $b=-1.5$ that can account for the relatively flat spectrum of the source (Chatterjee et al. 2017). The peak Lorentz factor of the distribution, $\gamma_{\max }$, is chosen to correspond to the observed spectral break frequency of $v_{\max }=10 \mathrm{GHz}$. If the lower Lorentz factor cut-off corresponds to emission at $v_{\min }=1 \mathrm{GHz},{ }^{4}$ then the equipartition magnetic field and electron column thus determined for minimum and maximum source sizes are $B_{\mathrm{eq}} \approx 140 \mathrm{mG}$, $\gamma_{\text {min }} \approx 50, \gamma_{\text {max }} \approx 160, N_{\text {rel }} \approx 0.95 \mathrm{pc} \mathrm{cm}^{-3}$ for $R_{\mathrm{per}}=0.05 \mathrm{pc}$, and $B_{\mathrm{eq}} \approx 27 \mathrm{mG}, \gamma_{\min } \approx 120, \gamma_{\max } \approx 370, N_{\text {rel }} \approx 0.1 \mathrm{pc} \mathrm{cm}^{-3}$ for $R_{\text {per }}=0.35 \mathrm{pc}$. The reader can scale the equipartition field to other source sizes using $B_{\text {eq }}(R) \propto R^{-6 / 7}$. The total energy contained in the relativistic electrons and the magnetic field ('equipartition energy') is $\sim 10^{49.1}$ and $\sim 10^{50.2} \mathrm{erg}$, respectively. If the relativistic electrons were injected in a one-off event, the synchrotron cooling rates at $\gamma_{\max }$ yield source ages of $14 \mathrm{yr}$ for $R=0.05 \mathrm{pc}$ and $60 \mathrm{yr}$ for $R=0.35 \mathrm{pc}$. The corresponding expansion velocities are $0.011 \mathrm{c}$ and $0.02 c$, respectively.

\section{FARADAY CONVERSION}

If the FRB progenitor resides within the persistent source, then relativistic corrections to the effects of birefringence must be accounted for in order to accurately determine the levels of Faraday rotation and conversion (collectively called generalized Faraday rotation) in the persistent source. The effect is readily visualized on the Poincaré sphere as the rotation of the polarization vector about an axis defined by the natural modes in the medium (see Kennett \& Melrose 1998, for details). Two conditions must be satisfied to attain appreciable conversion of linear to circular polarization due to propagation effects: (a) the natural modes in the medium must be sufficiently elliptical as characterized by their axial ratios, and (b) there must be a sufficient magneto-ionic column for this elliptical birefringence to have a measurable effect. In Fig. 1, we plot the level of generalized Faraday conversion for two commonly encountered electron distributions: a relativistic Maxwellian and a power law with an assumed index $b=-1.5$. We use the approximate expressions of Huang \& Shcherbakov (2011, their equations 51, 58, and 59) to do so. The upper panels show the peak circular fraction allowed by the ellipticity of the natural modes [condition (a) above] and the bottom panels show the generalized Faraday rotation angle [condition (b) above]. The plots assume $v=4 \mathrm{GHz}$, $\theta=\pi / 4$ (giving $\eta_{B} \approx 0.707$ ), and are normalized to a total electron column of $1 \mathrm{pc} \mathrm{cm}^{-3}$. It is worth noting that in the power law case, the mode ellipticity goes from the cold plasma limit (circular modes) to its ultrarelativistic limit (linear modes) in a rather narrow range of $2 \lesssim \gamma_{\text {min }} \lesssim 20$. Faraday conversion therefore gives us access to electrons in this range that cannot be studied by canonical means (via synchrotron emission for example).

For 'one-zone' models where the synchrotron emission and Faraday rotation come from the same nebula, condition (b) above is satisfied by definition and condition (a) must be reconciled with the non-detection of circular polarized emission. If the electron energies are power-law distributed, then Fig. 1 (top right-hand panel) shows that for $B=30,100$, and $250 \mathrm{G}$, the circular fraction is in tension with observations for $\gamma_{\min }>3.6,2.3$, and 1.7, respectively. The one-zone nebula must therefore be an admixture of synchrotron electrons $(50 \lesssim \gamma \lesssim 370)$ and 'cold' plasma $(\gamma \lesssim 3)$.

${ }^{4}$ This assumption will be relaxed in Section 3 .
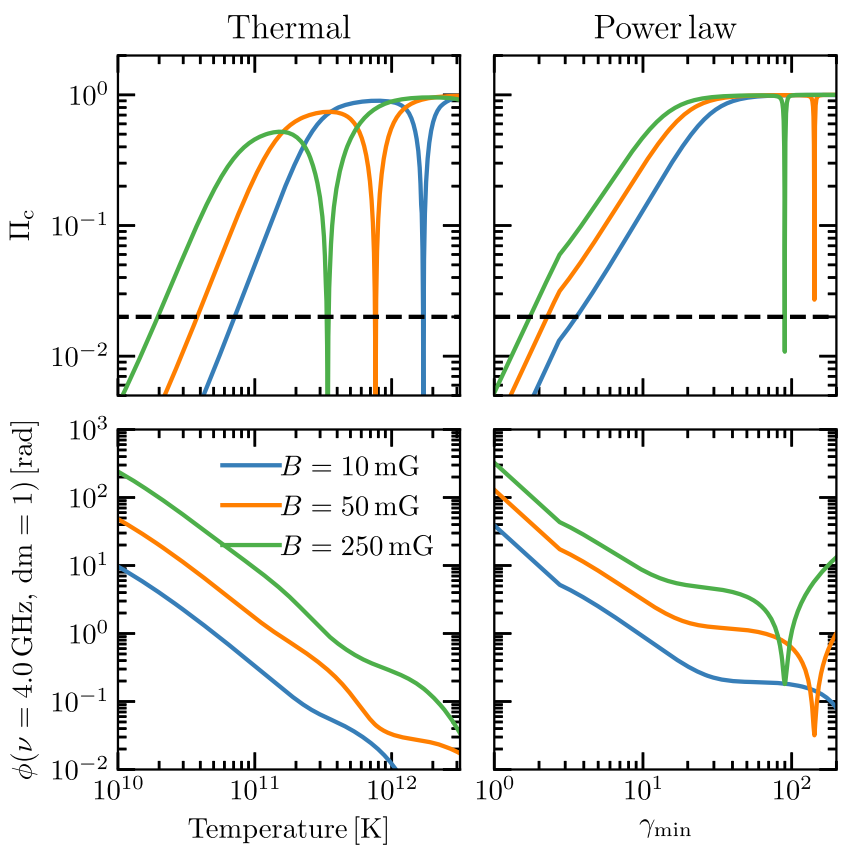

Figure 1. The effect of elliptical birefringence at $v=4 \mathrm{GHz}$ for thermal (left-hand panels) and power-law electron populations (right-hand panels; energy index of -1.5 ), for three different magnetic field values (different line colours). Top panels show the peak circular fraction, and bottom panels show the phase angle associated with generalized Faraday rotation. An electron column of $N_{\mathrm{e}}=1 \mathrm{pc} \mathrm{cm}^{-3}$ (which is $1 \mathrm{DM}$ units) has been assumed in all plots. Dashed black line in the top panels is placed at 2 per cent that corresponds to the observed upper limit on the circularly polarized fraction of FRB 121102 between 4 and $8 \mathrm{GHz}$ (Michilli et al. 2018). The deep notches in the plots are due to zero crossings.

For 'two-zone' models, the bulk of the observed Faraday rotation occurs outside the synchrotron source in a presumably cold plasma. If the radio bursts originate from within the synchrotron source, then the observed lack of circularly polarized radiation still requires condition (a) and (b) to not be satisfied simultaneously in the synchrotron source. Taking equipartition solutions for the persistent source from Section 2.3 with $v_{\min }=1 \mathrm{GHz}$, we find that the model is in tension with the observations for $R<0.31 \mathrm{pc}$. If we allow $\gamma_{\min }$ to correspond to $v_{\min }=100 \mathrm{MHz}$, then the equipartition solutions are in tension with polarimetric data over the entire feasible parameter range of $0.05<R / \mathrm{pc}<0.35$. However, by extending the energy distribution to $\gamma_{\min } \lesssim 3$ (and admixture of 'cold' and relativistic electrons), the modes can be constructed to be sufficiently circular so as to produce $<2$ per cent circular polarization as in the "onezone' case.

In summary, in all models where the radio bursts pass through the persistent source powered by a power-law electron energy distribution, the distribution must extend to $\gamma_{\min } \lesssim 3$, failing which (i) the Faraday screen cannot be co-located with the synchrotron emitting electrons, and (ii) the synchrotron source must have a rather fine-tuned radius in the narrow range $0.31<R / \mathrm{pc}<0.35$. If the electrons that are injected into the nebula are highly relativistic, then $\gamma_{\min }<3$ can be attained by radiative cooling over a time-scale of 275 and $7400 \mathrm{yr}$ for $R=0.05$ and $0.35 \mathrm{pc}$, respectively. If the electrons instead cool via adiabatic expansion from an injection Lorentz factor of $\gamma \gtrsim \gamma_{\max }$, then they must have been injected when the nebula was $<\left(9.4 \times 10^{-4}, 2.8 \times 10^{-3}\right)$ pc if the present size of the nebula is $(0.05,0.35) \mathrm{pc}$. These results can be directly applied to the one- 

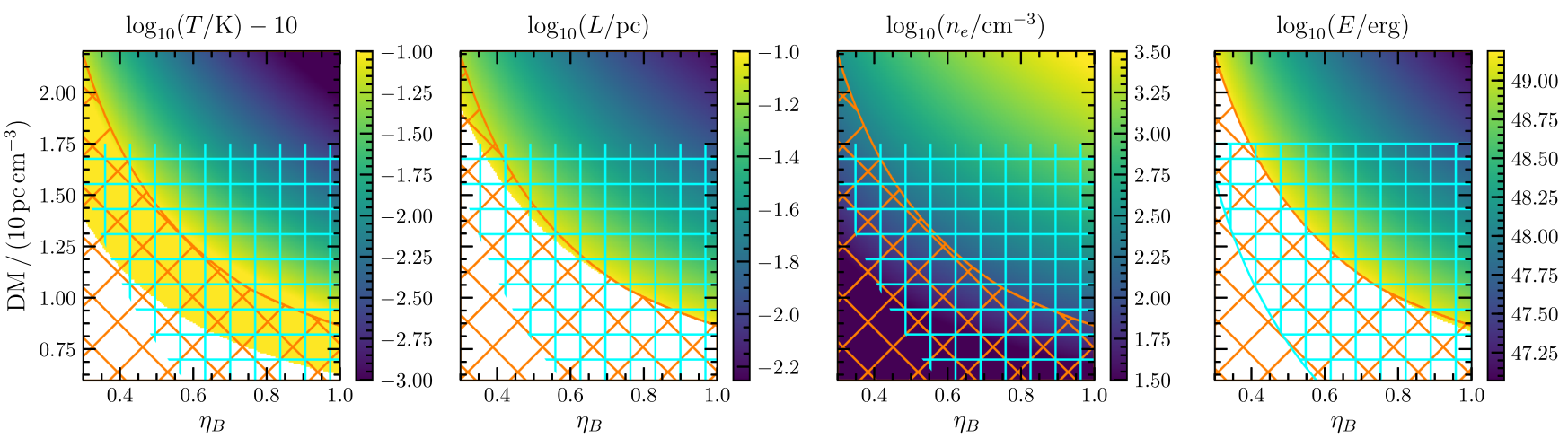

Figure 2. Constraints on the expanding nebula model of Waxman (2017) determined from equation (5), for plasma $\beta=1$ and persistent source radius of $R_{\text {per }}=0.1$. The colour map for each parameter is set to white when that parameter violates bounds described in Section 4.1. The cross-hatched (orange) region is excluded as it violates at least one bound. The straight-hatched region (cyan) is the allowed parameter range due to constraints from Faraday conversion and magneto-ionic variations (see Section 3).

zone magnetar model of Margalit \& Metzger (2018). Consider their benchmark model with $B=0.25 \mathrm{G}$, injection energy of $\gamma_{\text {inj }}=200$, energy distribution of $N_{\gamma} \propto \gamma^{-1.3}$ for $\gamma \leq \gamma_{\text {inj }}$, and nebular age of $\tau=12.4$ yr. The lack of observed circular polarization at $4 \mathrm{GHz}$ then requires $\gamma_{\min }<1.45$. If this is accomplished via adiabatic expansion, then the electron injection must have started when the central source was $12.4 \times 1.45 / 200=0.09$ yr. Faraday conversion constraints therefore require significant magnetic and baryonic flux to be ejected from the postulated magnetar within a month of its birth.

\section{DISCUSSION}

\subsection{Expanding relativistic nebula}

We consider the generic 'two-zone' model of Waxman (2017), where the source of FRB 121102 is a compact object centrally located in a synchrotron nebula, and the expansion of the synchrotron nebula is constrained by a much denser cold nebula. The Faraday rotation is provided by the shocked (and heated) part of the confining dense nebula. Notwithstanding the FRB generating mechanism and the nature of the compact object, this model links the velocity of the shock driven by the expanding synchrotron nebula into the surrounding colder medium and the density of the cold medium via $v_{\mathrm{sh}} \approx \sqrt{P_{\mathrm{sh}} /\left(n m_{\mathrm{p}}\right)}$, where $m_{\mathrm{p}}$ is the proton mass, and $P_{\mathrm{sh}}=B^{2}(1+\beta) /(8 \pi)$ is the pressure in the shocked part of the nebula that is similar to that in the synchrotron source. In convenient units, we have

$v_{\mathrm{sh}} \approx 9.1\left(\frac{T_{4}(1+\beta)}{\beta}\right)^{1 / 2} \mathrm{~km} \mathrm{~s}^{-1}$.

We now equate $v_{\text {sh }}$ with the expansion velocity in equation (3) to obtain the following family of models for the Faraday screen:

$$
\begin{aligned}
T_{4} & =2.94 \times 10^{4} \beta(1+\beta) \eta_{B}^{-4} \mathrm{DM}_{10}^{-6}, \\
l & =0.0323 \mathrm{DM}_{10}^{-3} \eta_{B}^{-2}(1+\beta) \mathrm{pc}, \\
n_{\mathrm{e}} & =316.3 \mathrm{DM}_{10}^{4} \eta_{B}^{2}(1+\beta)^{-1}, \\
E_{\mathrm{sh}} & =10^{49.2}\left(R_{\mathrm{per}} / \mathrm{pc}\right)^{2} \mathrm{DM}_{10}^{-5} \eta_{B}^{-4}(1+\beta)^{2} \mathrm{erg},
\end{aligned}
$$

where $E_{\mathrm{Sh}}$ is the combined thermal and magnetic energy in the shock-heated Faraday screen. A feasible model from the above family must additionally satisfy the following constraints. (i) To avoid violating Faraday conversion constraints, we need $T_{4}<10^{5} \mathrm{~K}$ (see Fig. 1, bottom left-hand panel). (ii) The Faraday-rotating plasma is presumably shock heated by the expanding relativistic gas, which requires the total energy in the latter to be larger than that in the former. (iii) As in Waxman (2017), we assume that the thickness of the Faraday screen must be smaller than the radius of the persistent source, $R_{\mathrm{per}}$. The constraints on the family of models are graphically shown in Fig. 2 for a benchmark value of $R_{\mathrm{per}}=0.1 \mathrm{pc}$ and $\beta=1$. We find feasible parameters ranges of $\mathrm{DM}_{10} \gtrsim 1.0$ and $\eta_{B} \gtrsim 0.4$ with a very weak dependence on $R_{\mathrm{per}}$. Taken together with the constraint of $\mathrm{DM}_{10}<1.75$ from Section 2.2, the allowed parameter ranges for the Faraday screen are tightly constrained: $\mathrm{DM}_{10} \in[1.0,1.75], \eta_{B} \gtrsim 0.4, B \eta_{B} \in[10,18] \mathrm{mG}$, and $\log _{10}(T / \mathrm{K}) \in[7.5,9]$. It is noteworthy and non-trivial that these self-consistent solutions should exist after a relationship between $v, T_{4}$, and $\beta$ due to equation (4) was imposed on observational constraints from equation (3).

\subsection{Dense filaments à la Crab}

Following the suggestion by Cordes et al. (2017) that FRB 121102 is lensed by dense plasma structures similar to the cold filaments in the Crab Nebula (Backer, Wong \& Valanju 2000), we consider a model where the variable RM and DM are obtained by transverse passage of dense filaments across the line of sight to the FRB source. Hessels et al. (2018, their section 4.4 and equations 6-9) have summarized the resulting constraints in terms of the peak frequency at which lensing is apparent $\left(v_{1}=8 \mathrm{GHz}\right)$, the source-lens distance $D_{\mathrm{sl}}$ (units of pc), and the observer-lens distance $D_{\mathrm{ol}} \approx D_{\mathrm{A}}=622 \mathrm{Mpc}$. Requiring the filament to have the similar transverse and line-ofsight extents, and to be transparent to free-free absorption at $1 \mathrm{GHz}$, we obtain the following:

$$
\begin{aligned}
T_{4} & >5.5 \mathrm{DM}_{10}^{1.15} D_{\mathrm{sl}}^{-0.385}, \\
n_{\mathrm{e}} & >2.7 \times 10^{6}\left(\frac{\mathrm{DM}_{10}}{D_{\mathrm{sl}}}\right)^{1 / 2} \mathrm{~cm}^{-3}, \\
\left(\frac{\eta_{B} T_{4}}{\beta}\right) & >1.7 D_{\mathrm{sl}}^{1 / 2} \mathrm{DM}_{10}^{-2.5}, \\
l & <3.7 \times 10^{-6}\left(D_{\mathrm{sl}} \mathrm{DM}_{10}\right)^{1 / 2} \mathrm{pc} .
\end{aligned}
$$

Anticipating large electron densities for filaments located within the persistent nebula $\left(d_{\mathrm{sl}}<1\right)$, we impose an additional constraint: the bremsstrahlung cooling time-scale, $\tau_{\mathrm{ff}}$, should exceed the duration over which the putative lensing has been observed. 
Although the bursts have only been observed since 2012, we conservatively assume $\tau_{\mathrm{ff}}>10 \mathrm{yr}$. Using Rybicki \& Lightman (1979, their equation 5.15b) the cooling time-scale is

$\tau_{\mathrm{ff}}=0.26 T_{4}^{1 / 2}\left(\frac{n_{\mathrm{e}}}{10^{6} \mathrm{~cm}^{-3}}\right)^{-1}>10$.

The simplest model we consider here is one where the Faraday rotation and lensing happens in the same filamentary structure/complex. To achieve this, we need plasma parameters that satisfy equations (3), (6), and (7) simultaneously. A scan through the parameter space shows that self-consistent solutions are only obtained for $\eta_{B}<0.2$. If we further require the putative filament to lie within the synchrotron nebula $\left(d_{\mathrm{ls}}<0.35 \mathrm{pc}\right.$, as defined by the persistent source), then $\eta_{B} \lesssim 10^{-4}$ that will lead to unrealistically large magnetic fields. We therefore conclude that the same filamentary complex cannot provide the postulated plasma lensing, and the observed Faraday rotation and variations thereof.

One could decouple Faraday-rotating and lensing plasma and readily find self-consistent solutions for two different plasma structures using equations (3) and (6) separately. For instance, lensing can be caused by structures with plasma parameters of $T_{4} \sim 10^{5}$, $\mathrm{DM}_{10} \sim 1$, and $n_{\mathrm{e}} \sim 10^{6} \mathrm{~cm}^{-3}$. The $\mathrm{RM}$ variations can be provided by transverse velocity of $\sim 30 \mathrm{~km} \mathrm{~s}^{-1}$ across a plasma structure with parameters of $T_{4} \sim 10^{2}, l \sim 2 \times 10^{-4} \mathrm{pc}$, and $n_{\mathrm{e}} \sim 5 \times 10^{4} \mathrm{~cm}^{-3}$.

\section{CONCLUSIONS}

We have shown that Faraday conversion of linear to circular polarized radiation is a relevant effect for FRBs that propagate through dense and relativistic magneto-ionic media. We have applied constraints from Faraday conversion and magneto-ionic variations to the test case of FRB 121102 by assuming a spatially homogeneous Faraday-rotating/converting medium (the orientation of the magnetic field is allowed to vary). We reach the following conclusions.

(i) If the radio bursts pass through the synchrotron nebula, then the latter must be an admixture of highly relativistic and cold electrons. Specifically, if the electron energies are power-law distributed, then they must extend to $\gamma_{\min } \lesssim 3$ in order to not violate Faraday conversion limits.

(ii) The 'one-zone' family of magnetar models posited by Margalit \& Metzger (2018) is only consistent with Faraday conversion constraints of the magnetic flux diffusion from the magnetar initiates almost immediately after its birth. For their benchmark model of $B=0.25 \mathrm{G}, N_{\gamma} \propto \gamma^{-1.3}, \gamma_{\mathrm{inj}}=200$, age of $12.4 \mathrm{yr}$, the onset must be within a month of magnetar's birth.

(iii) In models where the persistent source associated with FRB 121102 is confined by a colder Faraday-rotating plasma shell (e.g. Waxman 2017), the latter is required to be shock heated to $\sim 10^{7.5}-10^{9} \mathrm{~K}$, have an electron column of $10-17.5 \mathrm{pc} \mathrm{cm}^{-3}$, a geometric parameter of $0.4 \lesssim \eta_{B} \leq 1$, and magnetic field of $10<\eta_{B} B<18 \mathrm{mG}$. The existence of such a self-consistent and overconstrained solution is not trivial and lends credence to the model.

(iv) In models involving dense filaments as in the Crab Nebula, the magneto-ionic variations (DM and $\mathrm{RM}$ ) cannot come from the same plasma structures that also act as a plasma lens that is postulated to generate certain time-frequency structures seen in FRB 121102 (Cordes et al. 2017).

We emphasize that observations targeting the detection of Faraday-converted circular polarization at $\sim 1 \mathrm{GHz}$ in bursts from FRB 121102 are likely of great interest. We further advocate for 'derotation' of circular polarized signals in FRBs with linear polarization fractions below unity, in the event that Faraday-converted circular polarization has been averaged out. Finally, we anticipate that the arguments presented here will be of significance to other FRBs (particularly of a repeating nature) that are associated with radio-synchrotron sources and/or dense magneto-ionic media.

\section{ACKNOWLEDGEMENTS}

The authors thank (i) Andrei Gruzinov and Eli Waxman for discussions on the implications of Faraday conversion, (ii) Eli Waxman for commenting on the manuscript, (iii) Jason Hessels for discussions regarding observational aspects of FRB 121102, (iv) Dongzi Li for pointing out an error in our application of Faraday conversion in an earlier version of the manuscript, and (v) the organizers of the 2018 Schwartz/Reisman Institute for Theoretical Physics workshop on Fast Radio Bursts for their hospitality. Numerical computations used SCIPY, NUMPY, PYTHON2.7, and PYTHON3.0. MATPLOTLIB was used to render the figures.

\section{REFERENCES}

Backer D. C., Wong T., Valanju J., 2000, ApJ, 543, 740

Chatterjee S. et al., 2017, Nature, 541, 58

Cohen M. H., 1960, ApJ, 131, 664

Cordes J. M., Wasserman I., Hessels J. W. T., Lazio T. J. W., Chatterjee S., Wharton R. S., 2017, ApJ, 842, 35

Gruzinov A., Levin Y., 2019, ApJ, in press (arXiv:1902.01485)

Hessels J. W. T. et al., 2018, preprint (arXiv:1811.10748)

Homan D. C., Attridge J. M., Wardle J. F. C., 2001, ApJ, 556, 113

Huang L., Shcherbakov R. V., 2011, MNRAS, 416, 2574

Kennett M., Melrose D., 1998, Publ. Astron. Soc. Aust., 15, 211

Marcote B. et al., 2017, ApJ, 834, L8

Margalit B., Metzger B. D., 2018, ApJ, 868, L4

Masui K. et al., 2015, Nature, 528, 523

Michilli D. et al., 2018, Nature, 553, 182

Pacholczyk A. G., Swihart T. L., 1970, ApJ, 161, 415

Readhead A. C. S., 1994, ApJ, 426, 51

Rybicki G. B., Lightman A. P., 1979, Radiative Processes in Astrophysics.

Wiley-Interscience, New York

Sazonov V. N., 1969, SvA, 13, 396

Spitler L. G. et al., 2014, ApJ, 790, 101

Spitler L. G. et al., 2016, Nature, 531, 202

Tendulkar S. P. et al., 2017, ApJ, 834, L7

Walker M. A., 1998, MNRAS, 294, 307

Waxman E., 2017, ApJ, 842, 34

White S. M., Thejappa G., Kundu M. R., 1992, Sol. Phys., 138, 163

Zheleznyakov V. V., Zlotnik E. Y., 1964, SvA, 7, 485

This paper has been typeset from a $\mathrm{T}_{\mathrm{E}} \mathrm{X} / \mathrm{L} \mathrm{T}_{\mathrm{E}} \mathrm{X}$ file prepared by the author. 